\title{
Multivariate Analysis in Gas Sensing Applications
}

\author{
A. Szpakowski*, C. Tyszkiewicz and T. Pustelny \\ Institute of Physics, Department of Optoelectronics \\ Silesian University of Technology, Krzywoustego 2, 44-100 Gliwice, Poland \\ In gas sensing applications we often use sensors which have poor se- \\ lectivity. Such sensors are mature, cheap devices with simple application \\ circuits. Unfortunately, their cross sensitivity greatly restricts their useful- \\ ness when used in systems which employs univariate analysis. One method \\ which allows to overcome this difficulty is utilization of multivariate analysis \\ methods applied to the response from the group of such non-selective sensors. \\ This work presents the multivariate, pattern recognition system utilized to \\ process the response of an array of non-selective semiconductor sensors to \\ obtain the qualitative information.
}

PACS numbers: 02.50.Sk, 07.07.Df

\section{Introduction}

One of the most recognized multivariate technique is pattern recognition [1-3]. In short, the pattern recognition can be characterized as a process in which the raw data is collected and then processed based on the category to which the data belongs. Important feature of the system using the pattern recognition technique is that the input, sensor information, must be superfluous. In sensor applications this means that the sensor, or in most cases the array of sensors, must provide cross-selective information. The pattern recognition procedure is able to convert such information to usable ones.

In general, the pattern recognition procedure is composed of four steps: pre-processing, feature extraction, classification and identification.

In most cases, the aims of pre-processing is shifting, compressing and normalizing the raw signal from the sensors in order to improve the performance of the next steps. In same cases the signal is also filtered.

In the next step, the feature extraction is responsible for reducing the dimensionality of the output signal [4]. In most cases the sensor data in such systems is a time dependent data of sum transient state or states. In raw state such data

*corresponding author; e-mail: aszpakowski@polsl.pl 
has very high dimensionality, often in order of millions. To perform the analysis of such data, one must reduce the number of dimensions to hundreds or at most thousands. This reduction can be achieved in numerous ways using simple descriptors such as maximum/minimum or slope values or more advanced ones such as integral or differential features, features in phase space [5] or features extracted using wavelets decomposition [6].

Such reduced data are used in classification step. In this step the most important is a procedure that cluster together the features in classes, which mirror the chemical composition of sensor environment. This step can be quite extensive and be extended with further dimension reduction techniques working in union with feature space search techniques [7].

In the last step, the identification step, the signal from the array of sensors is assigned to a class and the occurrence of a particular pattern is recognized [1,2].

\section{Experimental setup}

The sensor array used in the experiment is composed of solid-state gas sensors based on $\mathrm{SnO}_{2}$. Their features important to our setup are high sensitivity, good accuracy, short response time, good stability and quite simple application circuits $[8,9]$. The major disadvantage of such sensors in most applications is their non-selectiveness and cross-sensitivity. Fortunately, in pattern recognition systems this feature is a desired one. In our setup we are using a group of eight FIGARO sensors, whose list is given in Table.

TABLE

Sensors forming the array and gases to which the sensors are destinated in compliance with data sheets provided by FIGARO Engineering Inc.

\begin{tabular}{c|l|l|l}
\hline \hline Sensor & \multicolumn{1}{|c|}{ Destination } & \multicolumn{1}{c}{ Sensor } & \multicolumn{1}{c}{ Destination } \\
\hline TGS800 & general air contaminations & TGS2602 & $\mathrm{C}_{3} \mathrm{H}_{8}, \mathrm{C}_{4} \mathrm{H}_{10}, \mathrm{CH}_{4}$ \\
TGS813 & $\mathrm{CH}_{4}, \mathrm{C}_{3} \mathrm{H}_{8}, \mathrm{C}_{4} \mathrm{H}_{10}$ & TGS2610 & $\mathrm{CH}_{4}, \mathrm{C}_{4} \mathrm{H}_{10}, \mathrm{H}_{2}$ \\
TGS842 & $\mathrm{H}_{2}, \mathrm{CO}$ & TGS2611 & $\mathrm{C}_{3} \mathrm{H}_{8}, \mathrm{C}_{4} \mathrm{H}_{10}, \mathrm{CH}_{4}$ \\
TGS2600 & general air contaminations & TGS2620 & $\mathrm{C}_{2} \mathrm{H}_{5} \mathrm{OH}, \mathrm{H}_{2}, \mathrm{C}_{4} \mathrm{H}_{10}$
\end{tabular}

The sensors were mounted in a chamber and worked in continuous flow controlled system in constant temperature mode. The time of exposition and purging periods were sufficient for achieving stationary response for each of the sensor. The measurements of the conductivity of the sensors were made automatically and the data were sampled with frequency $10 \mathrm{~Hz}$. The pre-processing procedure consists of logical analysis of signal from sensor array on the basis of signal from the gas controller in order to find the starting and ending points of adsorption/desorption cycles. Registered signal was filtered using the Hanning window. Finally, the base-line compensation for two modes, differential and proportional, was performed and the base-line conductivity of each of the sensors was calculated. 


\section{Experimental results}

The sensor array was exposed to cyclic interaction with different concentrations of hydrogen $\left(\mathrm{H}_{2}\right)$, methane $\left(\mathrm{CH}_{4}\right)$, carbon oxide $(\mathrm{CO})$, carbon dioxide $\left(\mathrm{CO}_{2}\right)$, ammonia $\left(\mathrm{NH}_{3}\right)$ and nitrogen dioxide $\left(\mathrm{NO}_{2}\right)$. All gases were diluted in synthetic air $(4 \% \mathrm{RH})$. In all experiments the total gas flow rate was $100 \mathrm{sccm}$. For each gas and concentration up to 70 features for each sensor were calculated which yields up to 560 dimensions in feature space. In feature extraction/selection phase this dataset was reduced to three dimensions. The reduction was performed using the feature subset selection (FSS) method based on filter approach. The employed search strategy was based on plus-l minus-r selection (LRS) [10], with independent component analysis (ICA) [11] as a dimensionality reduction tech-

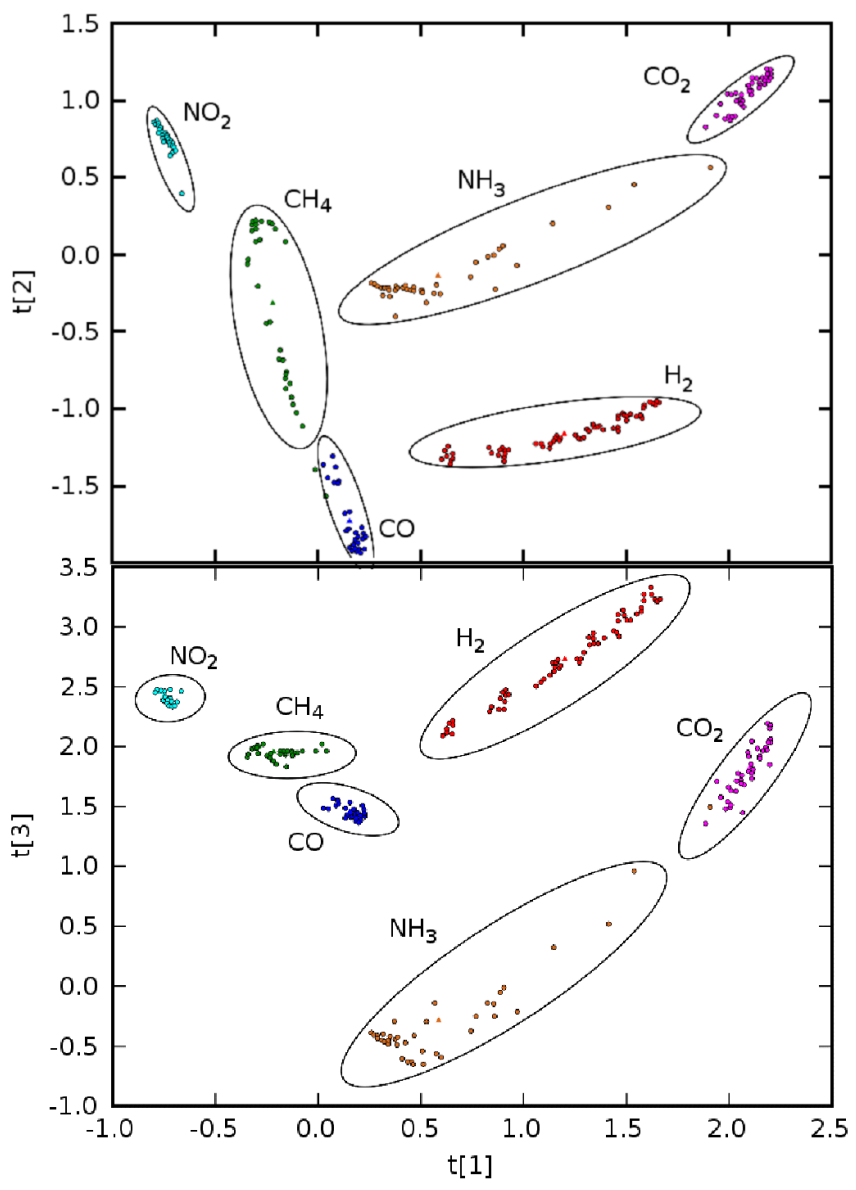

Fig. 1. Plots of the independent components in the secondaryfeature space show the good separation of sensors response to measured gases regardless of gas concentration. The upper and lower plots show the dependence of 1st vs. 2nd and 1st vs. 3rd independent component respectively. 
nique. In our program the JADE implementation of ICA algorithm was used [12]. As an evaluation method our own method was used. The method is based on the combination of scattering matrices, calculating variances of the distances of the cluster centers and employing the penalty functions. The method is strongly favoring the situation where clusters for each class (in our case for each gas) are evenly distributed and of even size. Additionally favored are situations where clusters are small and distant from each other. As a result, our FSS implementation was able to perform accurate separation of the six gases under consideration regardless of their concentration. The concentrations of the gases was respectively $\mathrm{H}_{2}$ : 0-20000 ppm, $\mathrm{NO}_{2}$ : 0-1850 ppm, $\mathrm{CH}_{4}: 0-500 \mathrm{ppm}, \mathrm{CO}: 0-500 \mathrm{ppm}, \mathrm{CO}_{2}$ : 0-5000 ppm, $\mathrm{NH}_{3}: 0-500 \mathrm{ppm}$. Results of application of presented method are shown in Fig. 1.

\section{Conclusions}

The employed pattern recognition methods allow us to achieve the separation of six gases using the array of eight, non-selective, general-purpose $\mathrm{SnO}_{2}$ sensors. Sensors were working in constant temperature mode in continuous flow chamber.

The future work involves using the sensors in temperature modulation mode, in closed chambers and in free air. Additionally, the numerical apparatus will be extended with classification and regression methods for classification of gas mixtures and evaluation of their concentrations.

\section{References}

[1] C. Di Natale, F. Davide, A. D'Amico, Sensors Actuators B 23, 111 (1995).

[2] R. Gutierrez-Osuna, IEEE Sensors J. 2, 189 (2002).

[3] R. Gutierrez-Osuna, H. Troy Nagle, IEEE Trans. Systems, Man, Cybernetics-Part B: Cybernetics 29, 626 (1999).

[4] Y. Yin, X. Tian, Sensors Actuators B 124, 393 (2007).

[5] E. Martinelli, C. Falconi, A. D'Amicoa, C. Di Natale, Sensors Actuators B 95, 132 (2003).

[6] Y. Tang, Frontiers Computer Sci. China 2, 268 (2008).

[7] E. Cantu-Paz, S. Newsam, C. Kamath, in: Proc. 10th ACM SIGKDD Int. Conf. on Knowledge Discovery and Data Mining, Seattle (WA, USA) 2004, Eds. W. Kim, R. Kohavi, J. Gehrke, W. DuMouchel, ACM Press, Seattle 2004, p. 788.

[8] W. Göpel, K.D. Schierbaum, Sensors Actuators B 26-27, 1 (1995).

[9] G. Korotcenkov, Mater. Sci. Eng. B 139, 1 (2007).

[10] A. Perera, T. Sundic, A. Pardo, R. Gutierrez-Osuna, S. Marco, Sensors J., IEEE 2, 235 (2002).

[11] L. De Lathauwer, B. De Moor, J. Vandewalle, J. Chemometrics 14, 123 (2000).

[12] J.F. Cardoso, A. Souloumiac, IEEE Proc. F 140, 362 (1993). 